\title{
Injectable Antiretroviral Drugs: Back to the Future
}

\author{
Marco Berruti ${ }^{1} \mathbb{D}$, Niccolò Riccardi ${ }^{2}$, Diana Canetti ${ }^{3,4}\left(\mathbb{D}\right.$, Sergio Lo Caputo ${ }^{5}$, Lucia Taramasso ${ }^{6}(\mathbb{D}$ and \\ Antonio Di Biagio 1,6,*
}

check for

updates

Citation: Berruti, M.; Riccardi, N.;

Canetti, D.; Lo Caputo, S.; Taramasso,

L.; Di Biagio, A. Injectable

Antiretroviral Drugs: Back to the

Future. Viruses 2021, 13, 228.

https://doi.org/10.3390/v13020228

Academic Editor: Julien Van

Grevenynghe

Received: 24 November 2020

Accepted: 25 January 2021

Published: 2 February 2021

Publisher's Note: MDPI stays neutral with regard to jurisdictional claims in published maps and institutional affiliations.

Copyright: (C) 2021 by the authors Licensee MDPI, Basel, Switzerland. This article is an open access article distributed under the terms and conditions of the Creative Commons Attribution (CC BY) license (https:/ / creativecommons.org/licenses/by/ $4.0 /)$.
1 Infectious Diseases Unit, Department of Health Sciences (DISSAL), University of Genoa, 16132 Genoa, Italy; marco.berruti1@gmail.com

2 Department of Infectious-Tropical Diseases and Microbiology, IRCCS Sacro Cuore Don Calabria Hospital, Negrar di Valpolicella, 37024 Verona, Italy; niccolo.riccardi@sacrocuore.it

3 Clinic of Infectious Diseases, IRCCS San Raffaele Scientific Institute, 20097 Milan, Italy; canetti.diana@hsr.it

4 School of Medicine, Vita-Salute San Raffaele University, 20097 Milan, Italy

5 Clinic of Infectious Diseases, Department of Clinical and Experimental Medicine, University of Foggia, 71122 Foggia, Italy; sergiolocaputo@gmail.com

6 Infectious Diseases Unit, Department of Internal Medicine, Ospedale Policlinico San Martino IRCCS, 16132 Genoa, Italy; taramasso.lucia@gmail.com

* Correspondence: adibiagioa@gmail.com

\begin{abstract}
Current HIV treatment regimens provide sustained virologic suppression, at least partially restore the immune system and have limited side effects; however, they do not allow viral eradication and they are burdened by daily pill intake with a life-long commitment for the people living with HIV (PHIV). Injectable agents might represent a turning point in the care of PHIV, allowing less frequent administration of antiretroviral treatment (ART), more widespread use of pre-exposure prophylaxis (PrEP) and more stable drug levels in the blood, thus increasing the odds to get closer to end the HIV pandemic. The aim of this manuscript is to give a comprehensive review of injectable antiretrovirals that have been used in the past, which are available now, will be available in the future, and their role in the treatment of HIV infection
\end{abstract}

Keywords: ART; injectable; HIV; antiretroviral; treatment; PrEP

\section{Introduction}

About 40 years have passed since the first cases of Acquired Immune Deficiency Syndrome (AIDS) were observed, and the HIV pandemic is still responsible for roughly 38 million people living with the virus and 1.7 million new infections every year [1,2]. Current treatments provide successful virological suppression, at least partially restore immune system and have limited side effects; however, they do not allow viral eradication and they are burdened by daily pill intake with a life-long commitment for the patients [3]. Moreover, in some countries with high HIV prevalence, like in Sub-Saharan Africa, difficulties in anti-retroviral treatment (ART) delivery and poor adherence pose a constant threat to achieve the 90-90-90 World Health Organization (WHO) strategy goals [4,5]. Furthermore, the emergence of multi-drug resistant (MDR) strains can favor virological failure, disease progression, and transmission [6]. Even if only an efficacious and free vaccine may be the answer for HIV eradication, long-acting injectable antiretrovirals may constitute a turning point for better infection control in difficult settings and decrease the emergence of resistance $[7,8]$. In fact, long-acting ART may improve adherence to therapy and extend prophylactic interventions as pre-exposure prophylaxis (PrEP) to vulnerable populations [9]. The aim of this manuscript is to give a comprehensive review of injectable antiretrovirals that have been used in the past, which are available now, will be available in the future, and their role in the treatment of HIV infection. 


\section{Methods}

We performed a MEDLINE/PubMed search on 03/03/2019 and the complete search string was as follows: (((injectable) AND antiretroviral) AND HIV) AND (Clinical trial) AND (“01/01/1990" (Date-Publication): “03/03/2019" (Date-Publication)). The string was then updated on the 30 June 2020 to enclose more recent papers

Of the 361 papers identified, 299 were excluded by title and abstract screening as they were not pertinent to our narrative review. We ultimately selected 36 papers: the full texts and pertinent references were then retrieved and collectively discussed. The decision about inclusion in the present narrative review was ultimately made according to the subjective impression of the authors. Eventually, the review was organized for each drug analyzed in the following paragraphs: (i) "Molecular characteristics"; (ii) "Available formulations"; (iii) "Recommended doses"; and (iv) "Place in therapy". Finally, a discussion about the future applications of the reviewed drugs is given.

\section{Zidovudine (AZT)}

\subsection{Molecular Characteristics}

Zidovudine (AZT) (3'-azido-2'3'-dideoxythymidine) was the first antiretroviral drug available in the 1980s. Initially studied as an antineoplastic drug, it subsequently showed in vitro and in vivo activity against HIV-1 virus and, in a lesser extent, against HTLV-1 and HIV-2. AZT is a thymidine analogue which intracellularly is phosphorylated to its active $5^{\prime}$-triphosphate metabolite, zidovudine triphosphate (ZDV-TP). The principal mode of action of ZDV-TP is the inhibition of reverse transcriptase (RT) via DNA chain termination after incorporation of the nucleotide analogue. ZDV-TP is a weak inhibitor of the cellular DNA polymerases $\alpha$ and $\gamma$ and has been reported to be incorporated into the DNA of cells in culture $[10,11]$ (Figure 1).

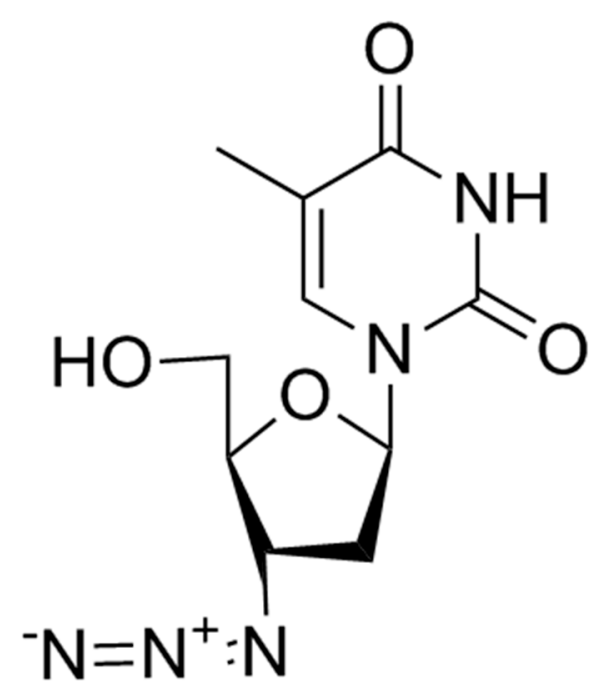

Figure 1. Zidovudine.

\subsection{Available Formulations}

AZT is available in both oral and parenteral formulation and, by a pharmacokinetic point of view, it shows good oral bioavailability $(64 \%)$ with serum concentration peak 30 to $90 \mathrm{~min}$ after administration [12]. The AZT serum half-life is $1 \mathrm{~h}$ but the intracellular half-life is about $3 \mathrm{~h}$ and is eliminated with urine after undergoing glucuronidation through the liver. Of note, AZT good distribution volume allows good penetration in the central nervous system (CNS) and through the placenta [12]. 


\subsection{Recommended Doses}

AZT is recommended at the dosage of $300 \mathrm{mg}$ every $12 \mathrm{~h}$ in oral formulation and $1 \mathrm{mg} / \mathrm{kg} /$ dose every $4 \mathrm{~h}$ in the prevention of perinatal transmission of HIV $(2 \mathrm{mg} / \mathrm{kg}$ loading dose followed by continuous infusion of $1 \mathrm{mg} / \mathrm{kg}$ until delivery).

\subsection{Place in Therapy}

AZT use is no longer recommended in the treatment of HIV infection; the role is limited to prevent mother-to child transmission: (a) intrapartum in women with HIV-RNA $>50$ copies $/ \mathrm{mL}$, and (b) in infants of HIV-infected mothers $[13,14]$ and as deep salvage treatment in patients with proven sensitivity at the genotypic resistance test [15]. Side effects, in particular severe anemia, gastrointestinal toxicity and early viral resistance, were the main barriers to usage.

\section{Enfuvirtide}

\subsection{Molecular Characteristics}

Enfuvirtide (T-20) was the first antiretroviral drug targeting viral entry process; it is a 36-aminoacid polypeptide derived from heptad repeat (HR) 2 that binds the HR-1 region of gp41 preventing the coil-coil zipping reaction and subsequently HIV-1 fusion with cellular membrane [16]. T-20, due to its peculiar mechanism of action, is active on both CXCR4-tropic and CCR5-tropic virus (Figure 2).

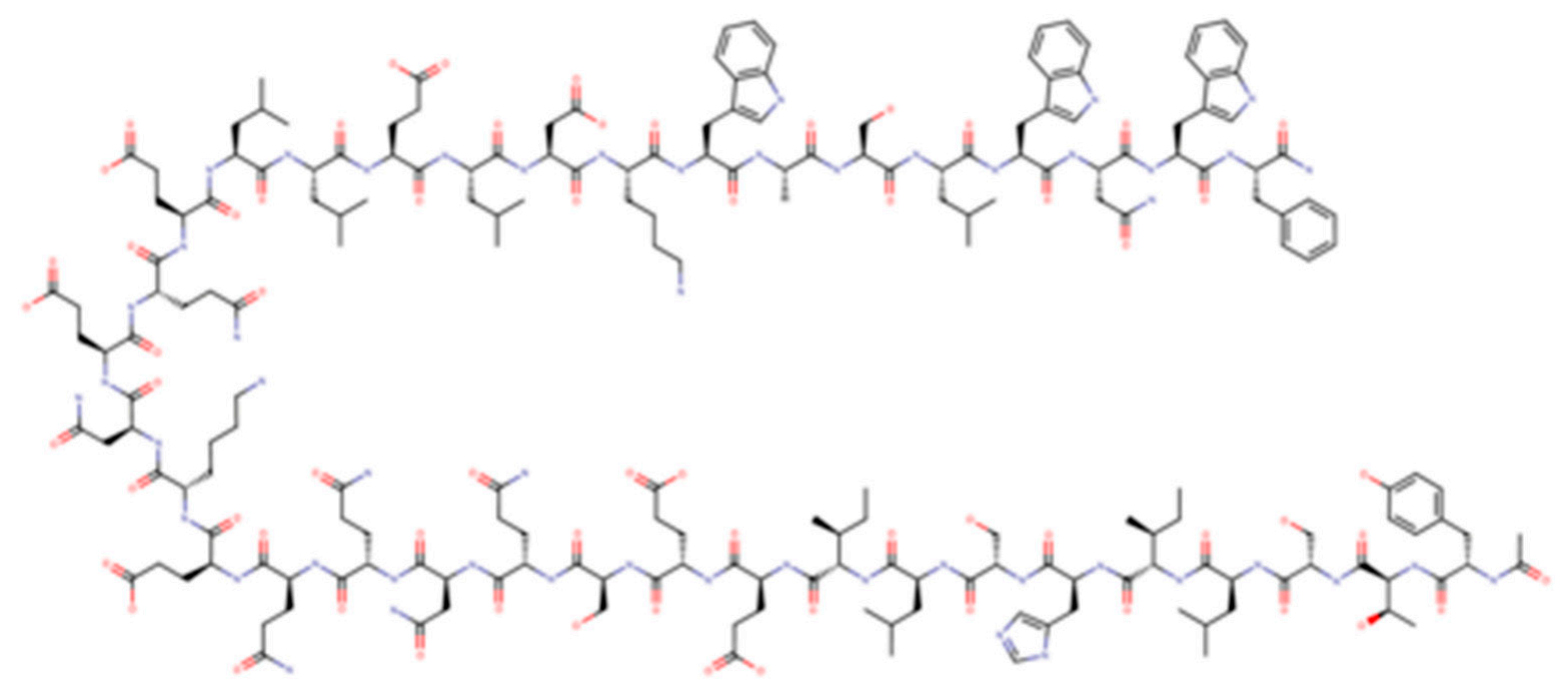

Figure 2. Enfuvirtide.

\subsection{Available Formulations}

This drug is available only in a subcutaneous compound due to its susceptibility to intestinal peptidases. T-20 has a concentration peak at $4 \mathrm{~h}$ and a plasmatic half-life ranging from 2 to $3.8 \mathrm{~h}$ without influence by renal or hepatic function. There are frequent adverse drug reactions, mainly cutaneous in the injection site.

\subsection{Recommended Doses}

The dosage of T-20 is $90 \mathrm{mg}$ every $12 \mathrm{~h}$ by subcutaneous administration.

\subsection{Place in Therapy}

T-20 use is no longer recommended and limited to as deep salvage treatment in patients with previous treatment failures. In these individuals, the use is recommended in combination with an optimized background therapy $[17,18]$. 


\section{Rilpivirine (RPV; TMC278) Long Acting (RPV-LA)}

\subsection{Molecular Characteristics}

Rilpivirine (RPV or previously TMC278) is a potent diarylpyrimidine NNRTI currently used for the treatment of ART-naïve patients with a CD4+ T cell count more than 200 cells $/ \mathrm{mm}^{3}$ and HIV-1 RNA levels less than 100,000 copies/mL and of ART-experienced patients without resistance mutations to RPV (Figure 3).<smiles>Cc1cc(/C=C/C#N)cc(C)c1Nc1ccnc(Nc2ccc(C#N)cc2)n1</smiles>

Figure 3. Rilpivirine.

\subsection{Available Formulations}

It is available in oral formulation ( $25 \mathrm{mg}$ once daily) as an individual drug preparation or as a single-tablet regimen (STR) in combination with tenofovir disoproxil fumarate or tenofovir alafenamide and emtricitabine (TDF/FTC/RPV; TAF/FTC/RPV) or with dolutegravir (RPV/DTG).

In oral formulation, RPV showed a good tolerability and a good pharmacokinetic (PK) profile. RPV should be taken with a normal caloric meal ( $533 \mathrm{Kcal}$ ) or high-fat high-caloric meal $(928 \mathrm{Kcal})$ and need careful evaluation of possible drug interactions, in particular with proton pump inhibitors and antiepileptic drugs. In a trial on healthy volunteers that were exposed to intravenous nanosuspension, maximum concentration was achieved in three days and decreased to below $10 \mathrm{ng} / \mathrm{mL}$ after 12-26 weeks with a half-life of five weeks [19-21].

\subsection{Recommended Doses}

In animal models, RPV, formulated as a $200 \mathrm{~nm}$ nanosuspension and administrated intramuscularly (IM) or subcutaneously (SC), showed sustained and dose-proportional release over 2-6 months with an absolute bioavailability of $100 \%$ in spite of high concentrations at the injection site. IM administration showed a higher peak concentration compared to SC. This study showed that nanoparticles generated a secondary depot of RPV by macrophage in the lymph nodes with an initial concentration 100-fold higher than plasma one month after the administration, which decreased 3-6 folds below plasma concentration beyond three months [22].

Long-acting RPV (RPV-LA) was also evaluated in a phase I study with a single injection of escalating doses and a phase II study with $1200 \mathrm{mg}$ loading dose followed by $600 \mathrm{mg}$ every four weeks. RPV was detected after 4 and $8 \mathrm{~h}$ after administration; analysis of plasma concentrations was performed up to 28 days after injection or up to $<20 \mathrm{ng} / \mathrm{mL}$. After a 300-, 600- or 1200-mg injection, RPV-LA PK was largely comparable to the 1200-mg loading dose and both 600-mg RPV-LA injections. The mean plasma concentration of RPV when administered every 28 days after the last injection in all dosing groups was $79 \mathrm{ng} / \mathrm{mL}$ [23]. 
Clinical Trials with Cabotegravir (CAB-LA)

1. The LATTE-2 study evaluated cabotegravir and RPV-LA in maintaining HIV-1 viral suppression for 96 weeks. In the randomized, phase $2 \mathrm{~b}$, open-label study, HIV-1-treated adults initially received oral cabotegravir $(\mathrm{CAB})$ plus $\mathrm{ABC} / 3 \mathrm{TC}$ once daily. The objective of this study was to select an effective, tolerable, and safe intramuscular dosing regimen of the two drugs compared with oral CAB plus ABC/3TC. After a 20-week induction period, patients with HIV- 1 RNA $<50$ copies per $m L$ were randomly assigned (2:2:1) to therapy with CAB LA + RPV-LA at 4-week intervals or at 8-week intervals or to continue oral CAB plus $\mathrm{ABC} / 3 \mathrm{TC}$. The primary endpoints were the proportion of patients with viral suppression at week 32, protocol-defined virologic failures, and safety events for 96 weeks. A total of 309 patients were enrolled, 286 were randomly assigned to the maintenance period (115 to each of the 4- and 8-week groups and 56 to the oral treatment group). At week 96, viral suppression was maintained in $47(84 \%)$ of 56 patients receiving oral treatment, $100(87 \%)$ of 115 patients in the 4-week group, and $108(94 \%)$ of 115 patients in the 8-week group. Three patients (1\%) had virological failure (two in the 8-week group; one in the oral treatment group). Injection site reactions were mild (3648 (84\%) of 4360 injections) or moderate (673 (15\%) of 4360 injections) and rarely led to discontinuation (two $(<1 \%)$ of 230 patients); injection site pain was most frequently reported. Serious adverse events during maintenance were reported in $22(10 \%)$ of 230 patients in the intramuscular groups (4- and 8-week groups) and in seven (13\%) of 56 patients in the oral treatment group; none were drug-related. In this study, the combination of CAB-LA and RPV-LA every four weeks or every eight weeks was as effective as daily three-drug oral therapy in maintaining HIV-1 viral suppression for 96 weeks and was well accepted and tolerated [24].

2. In a phase 3, randomized, open-label trial ART-treatment, naïve patients were given 20 weeks of daily oral induction therapy with DTG-abacavir (ABC)-lamivudine (3TC) (DTG/ABC/3TC). Participants who had an HIV-1 RNA $<50$ copies/mL after 16 weeks were randomly assigned (1:1) to continue the DTG/ABC/3TC or switch to oral cabotegravir plus RPV for one month followed by monthly injections of long-acting cabotegravir plus RPV. The primary end point was the percentage of participants who had an HIV-1 RNA $>50$ copies $/ \mathrm{mL}$ or more at week 48 (Food and Drug Administration snapshot algorithm). At week 48, an HIV-1 RNA $>50$ copies/mL or more was found in 6 of 283 participants (2.1\%) who received LA therapy and in 7 of $283(2.5 \%)$ who received oral therapy. This exciting result met the noninferiority criterion for the primary end point (margin, six percentage points). Regarding adverse reactions, in the group of participants who received LA, $86 \%$ reported injection site reactions (median duration, 3 days; mild or moderate severity, $99 \%$ of cases); 4 participants withdrew from the trial for injection-related reasons. Treatment satisfaction increased after participants switched to LA therapy; $91 \%$ preferred long-acting therapy at week 48 [25].

3. In a phase 3 , open-label, multicenter, noninferiority study involving patients with HIV-RNA $<50$ copies $/ \mathrm{mL}$ for at least six months while taking oral ART, they were randomized into two groups (1:1) to continue their oral therapy or switch to RPV-LA and cabotegravir-LA. The primary end point was the percentage of participants with an HIV-1 RNA level of 50 copies/mL or greater at week 48 .

Treatment was initiated in 308 participants per group. At week 48, HIV-1 RNA levels of 50 copies $/ \mathrm{mL}$ or more were detected in five participants $(1.6 \%)$ on long-acting therapy and in three $(1.0 \%)$ on oral therapy (adjusted difference, 0.6 percentage points; $95 \%$ confidence interval $[\mathrm{CI}],-1.2$ to 2.5$)$, a result that met the noninferiority criterion for the primary end point (noninferiority margin, six percentage points). Adverse events were more common in the long-acting group and included injection pain, which occurred in 231 patients (75\%), however, mild or moderate in most cases; and 1\% withdrew because of this event [26]. 


\subsection{Place in Therapy}

The combination of RPV-LA and CAB-LA is recommended for maintenance treatment of HIV-infected adults who have HIV-RNA $<50$ copies/mL, and when the virus has not developed resistance to NNRTIs and integrase strand transfer inhibitor (INIs).

\subsection{Place in Pre-Exposure Prophylaxis}

RPV LA IM injections every eight weeks in the HPTN 076 study were safe and acceptable. Overall, despite more injection site reactions and pain in the participants receiving RPV LA, the injections were well tolerated. Data from this study support the further development of injectable PrEP agents [27].

\section{Cabotegravir (CAB, GSK 1265744)}

Molecular Characteristics and Available Formulations

Cabotegravir (CAB) is an integrase strand transfer inhibitor (InSTI) similar to DTG that was initially studied in oral formulation (Figure 4). Then, it was made available in subcutaneous and intramuscular formulations using nanotechnology, achieving a long half-life, which allows for a monthly or quarterly dosing schedule with a good safety profile [28].

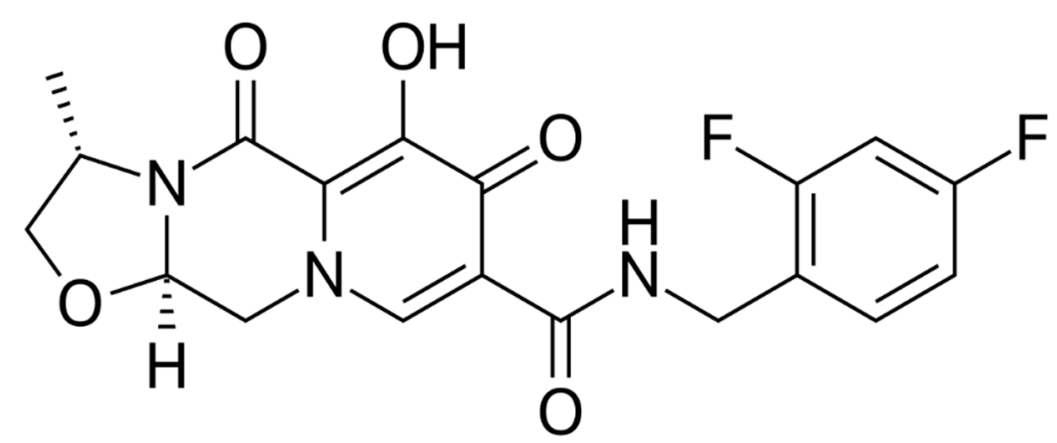

Figure 4. Cabotegravir.

\section{Clinical Trials with RPV (RPV-LA)}

See studies previously described in RPV-LA

\subsection{Place in Therapy}

The combination of RPV-LA and CAB-LA is recommended for maintenance treatment of HIV-infected adults who have HIV-RNA $<50$ copies $/ \mathrm{mL}$, and when the virus has not developed resistance to NNRTIs and InSTIs.

\subsection{Place in Pre-Exposure Prophylaxis}

Recently, the HIV Prevention Trials Network study (HPTN 084) on the safety and efficacy of CAB LA for pre-exposure prophylaxis (PrEP) in HIV-uninfected women, was stopped early by the trial Data and Safety Monitoring Board (DSMB) as results showed CAB LA to be highly effective in preventing HIV acquisition [29].

\section{Ibalizumab}

\subsection{Molecular Characteristics}

Ibalizumab (IBA) (Trogarzo ${ }^{\mathrm{TM}}$ ), formerly known as TNX-355, is a humanized IgG4 monoclonal antibody (MAb), which acts as an entry inhibitor. It binds the CD4 receptor, leading to its conformational change and interfering with its binding with HIV gp120 [30,31] (Figure 5). 

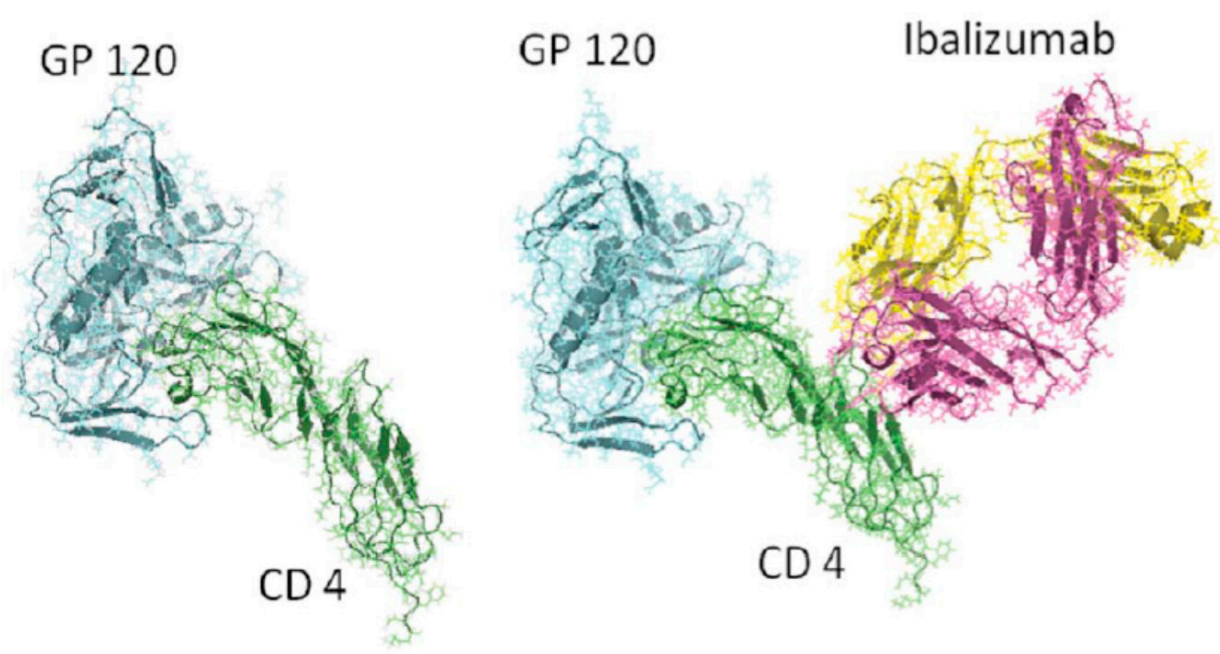

Figure 5. Ibalizumab.

\subsection{Available Formulations}

IBA is currently available as an intravenous infusion and, given its long half-life (3-3.5 days after both intravenous and subcutaneous administration), it can be administered every two weeks as a single loading dose of $2000 \mathrm{mg}$, followed by a maintenance dose of $800 \mathrm{mg}$.

This first biologic drug was studied for more than a decade with phase I and II trials demonstrating its efficacy and good tolerability profile.

\subsection{Recommended Doses}

In a single-group, open label, phase 3 study, 40 patients with an HIV-RNA higher than 1000 copies /mL, ART-treated for more than six months with a 3-class resistance were enrolled. The primary endpoint was the proportion of PLWH with a decrease in HIV-RNA load of at least $0.5 \log _{10}$ copies/mL from baseline (day 14). A total of 31 PLWH completed the study. Of the 40 PLWH in the ITT population, $33(83 \%)$ had a decrease in HIV-RNA load of at least $0.5 \log _{10}$ copies $/ \mathrm{mL}$ from baseline. At week 25 , a mean VL decrease of $1.6 \log _{10}$ from baseline was observed. Undetectable VL was observed in $43 \%$ of patients, while $50 \%$ of patients had a VL $<200$ copies/mL. The most common adverse events were diarrhea; 4 patients died from causes related to underlying illnesses while 1 patient developed an immune reconstitution syndrome [31].

\subsection{Place in Therapy}

IBA was approved by the FDA in 2018 and by the EMA on 26 September 2019 [32] in combination with other antiretroviral(s) and is indicated for the treatment of adults infected with multidrug resistant HIV-1 infection for whom it is otherwise not possible to construct a suppressive antiviral regimen.

\section{GS-6207}

\subsection{Molecular Characteristics}

GS-6207 is a first-in-class inhibitor of HIV-1 capsid function (CA) with a long-acting activity that can be administered monthly or less frequently in extended-release parenteral formulations thanks to its low aqueous solubility. It binds with high affinity to CA hexamers between adjacent CA monomers and retains sensibility against HIV-1 mutants resistant to other ARV classes including mutants with a Gag alteration that conferred resistance to other maturation agents (Figure 6). 


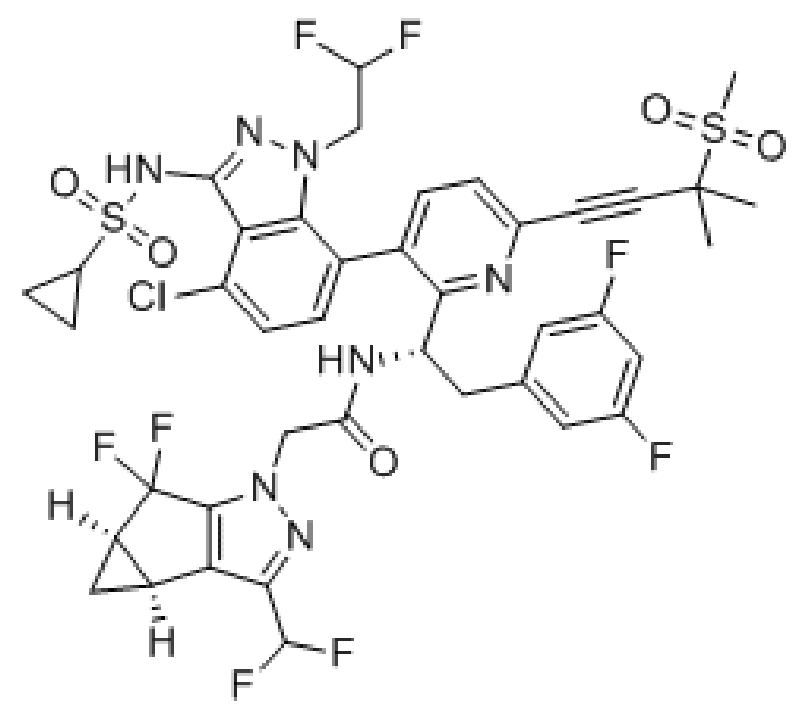

Figure 6. GS-6207.

In an in-vitro study, it displayed potent activity in MT-4 cells (EC50 $=0.1 \mathrm{nM}$, CC50 $=27 \mu \mathrm{M}$ ) and exhibited a mean EC50 of $0.05 \mathrm{nM}$ in human PBMCs against $23 \mathrm{HIV}-1$ clinical isolates including all subtypes. In the same study, GS-6207 human serum proteinadjusted EC95 was found to be $>10$-fold lower than that of efavirenz (EFV), RPV, DTG, and atazanavir (ATV) while in primary human CD4+ T-cells and macrophages was $>10$-fold more potent and $>22$-fold more selective than them. GS-6207 also suppressed HIV-2 replication. GS-6207 antiviral activity decreased with increasing multiplicity of infection (MOI), but remains 5- to $>100$-fold more potent than four commonly used ARVs and exhibits low cytotoxicity in four human cell lines and primary hepatocytes (CC50 $>44 \mu \mathrm{M})$. It also showed synergistic antiviral activity when combined with other ARV classes [33].

GS-6207 disrupts HIV capsid, a multimeric shell that is essential to viral replication, at multiple stages throughout the viral life cycle.

Results from a phase $1 \mathrm{~b}$ proof-of-concept study of a subcutaneous formulation showed antiviral activity with GS-6207 with significantly greater reductions in HIV-1 RNA versus the placebo across all treatment groups.

Preliminary PK data were consistent with sustained delivery with a dose interval of at least three months with a median apparent terminal $t_{1 / 2}$ between 30 to 38 days and concentrations measurable for at least 16 weeks. Tmax values ranged from 21 to 35 days and the increase in exposure $\left(\mathrm{C}_{\max }\right.$ and $\left.\mathrm{AUC}\right)$ between 30 and $100 \mathrm{mg}$ was proportionate to dose [34].

\subsection{Place in Therapy}

The above-mentioned data highlight the potential of GS-6207 for treatment in PLWH, regardless of ARV history.

\section{Albuvirtide (ABT)}

\subsection{Molecular Characteristics}

Albuvirtide (ABT) is a new injectable fusion inhibitor with once-a-week administration currently approved in China (Figure 7).

ABT is a 3-maleimidopropionic acid (MPA)-modified peptide HIV fusion inhibitor derived from the N-terminal sequence of HIV-1 gp41 which can irreversibly conjugate to serum albumin that extends its half-life. Unlike enfuvirtide, it manages to form a stable $\alpha$-helical conformation with the target sequence of gp41 and it blocks the fusion-active six-helix bundle (6-HB) formation in a dominant-negative manner preventing viral fusion and entry [35]. This mechanism of action permits potent inhibitory activity of ABT against a broad spectrum of HIV-1 strains including variants resistant to T20 [36]. 


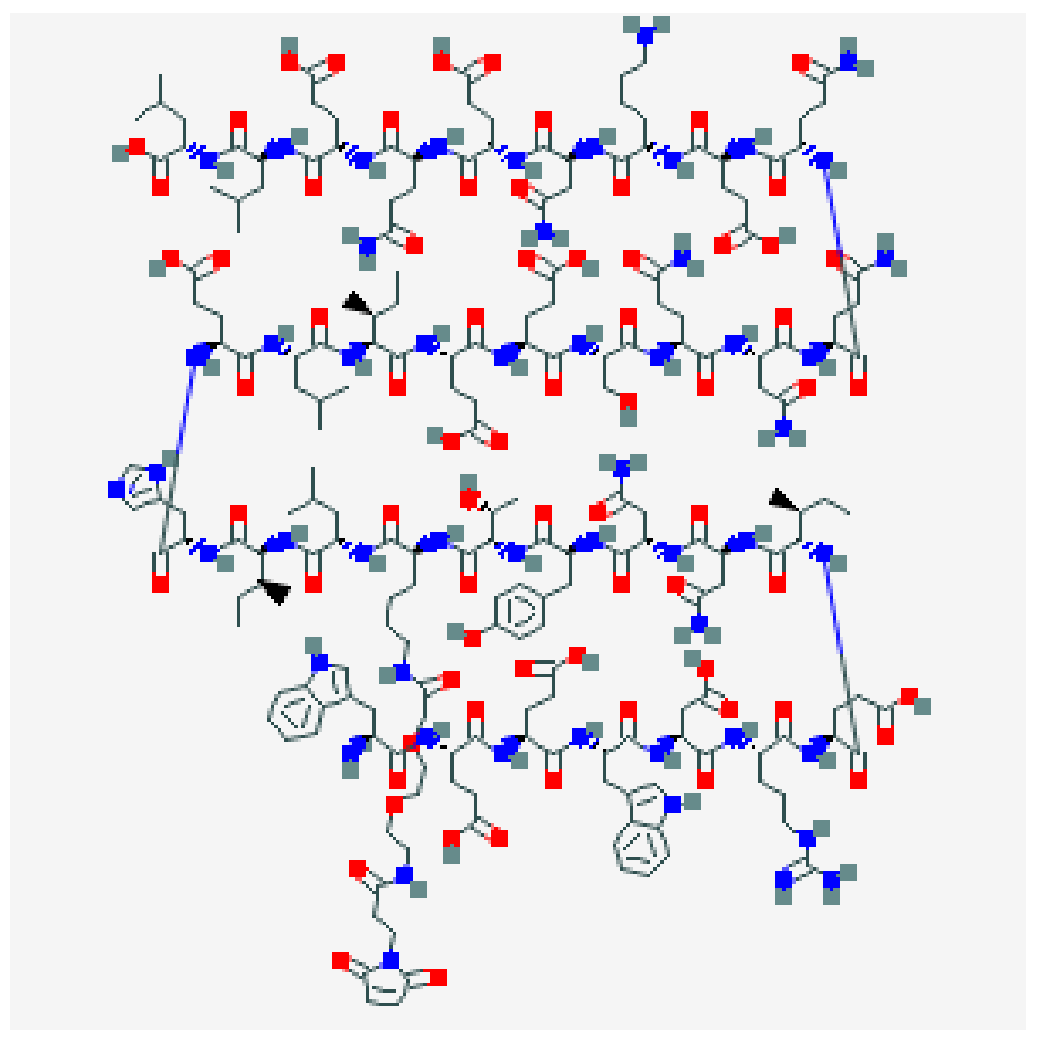

Figure 7. Albuvitirde.

This molecule was evaluated in vivo by Zhang et al. in an open-label and randomized trial on 20 ART naïve patients with a HIV-RNA > 5000 copies $/ \mathrm{mL}$ and a CD4+ cell count $>350$ cells $/ \mathrm{mm}^{3}$. They received either an intravenous weekly infusion of 160 or $320 \mathrm{mg}$ of ABT and lopinavir/ritonavir (LPV/r) 400/100 mg twice daily. At week 7, decline of 1.9 (1.3-2.3) $\log _{10}$ and 2.2 (1.6-2.7) $\log _{10}$ copies/mL of HIV-1 RNA was observed from the baseline. Viral load suppression (HIV-RNA < 50 copies $/ \mathrm{mL}$ ) was achieved in 11.1\% (1/9) and 55.6\% (5/9) patients, while the CD4+ cell count change was -5 cells $/ \mathrm{mm}^{3}$ and 52 for the 160 and $320 \mathrm{mg}$ dose group, respectively [37].

Results from the phase 3 multicenter, open-label, randomized, controlled, non-inferiority TALENT (Test ALbuvirtide in experiENced patienTs) in HIV-1 infected patients who failed ART were presented. Adults infected with HIV-1 were eligible for inclusion if they had a plasma viral RNA load of at least 1000 copies/mL or higher. Patients were allocated (1:1) to the ABT plus lopinavir boosted with the ritonavir (LPV/r) group or the LPV/r plus two optimized NRTIs groups were enrolled with 347 PLWH for 48 weeks. A HIV-RNA load $<50$ copies $/ \mathrm{mL}$ was obtained in $66 \%$ of the 2 NRTI group and $80 \%$ of the ABT group reaching the non-inferiority endpoint. Only in five patients in the ABT arm was a HIV-RNA load $>500$ copies $/ \mathrm{mL}$ detected without evidence of resistance. No death occurred in the two groups and the more common adverse events were gastrointestinal abnormalities, rash, headache, dizziness, hematuria, cholesterol, and triglyceride elevation with no significant differences between the two groups [38].

\subsection{Place in Therapy}

Currently, its role in therapy is still to be defined, potentially in heavily treatment experienced patients with limited treatment options left.

\section{1. bNAbs}

HIV-1-specific broadly neutralizing antibodies (bNAbs) may be a key player as an "add-on" agent for HIV prevention, treatment, and perhaps, eradication [39]. 
bNAbs have been shown to reduce VL and to potentially maintain viral suppression; however, rebound viremia with emergence of resistant acid strains has been observed in clinical trials as well as a not fully understood effect of the viral reservoir [39].

\section{Place in Therapy}

Although efficient neutralization of free HIV virus has been described, bNAbs need further investigations to find their proper place in therapy.

\section{Discussion and Conclusions}

In the last few years, much progress has been made in terms of antiretroviral therapy regimens with new drugs and new formulations, but HIV-resistant strains, ART interactions, and long-term ART adherence remain important issues [40-43].

The availability of routes of administration other than oral such as subcutaneous or intravenous may be an advantage and may allow the physicians to closely monitor assumption with direct observed therapy, especially in populations at high risk for interruptions (e.g., adolescents) or in PLWH who report drug fatigue. Long-acting drugs with administration every 1-2 or more months may effectively reduce poor adherence to treatment.

Pharmacokinetics and pharmacodynamics of injectable drugs allow more regular drug level through adequate therapeutic drug monitoring (TDM), decreasing the likelihood of erratic kinetics due to oral administration (e.g., with or without meals, gastric $\mathrm{pH}$ level), and a more stable antiretroviral effect in an optic of treat as prevention (TaSP) and PrEP.

Not the least, the access to molecules with a new mechanism of action opens awaited therapeutic chances to those heavily-experienced patients with few or no available treatment options (e.g., Ibalizumab).

In conclusion, injectable agents might represent a turning point in the care of people living with HIV, allowing less frequent administration of ART, and hopefully more widespread use of PrEP, more stable drug levels in the blood, thus increasing the odds to get closer to ending the HIV epidemic.

Funding: This research received no external funding.

Institutional Review Board Statement: Not applicable.

Informed Consent Statement: Not applicable.

Data Availability Statement: Data sharing not applicable.

Conflicts of Interest: All the authors declare no conflicts of interest.

\section{References}

1. Gottlieb, M.S.; Schroff, R.; Schanker, H.M.; Weisman, J.D.; Fan, P.T.; Wolf, R.A.; Saxon, A. Pneumocystis carinii pneumonia and mucosal candidiasis in previously healthy homosexual men: Evidence of a new acquired cellular immunodeficiency. N. Engl. J. Med. 1981, 305, 1425-1431. [CrossRef]

2. Unaids Data. 2020. Available online: https://www.unaids.org/sites/default/files/media_asset/UNAIDS_FactSheet_en.pdf (accessed on 30 June 2020).

3. Barré-Sinoussi, F.; Ross, A.L.; Delfraissy, J.-F. Past, present and future: 30 years of HIV research. Nat. Rev. Genet. 2013, 11, 877-883. [CrossRef] [PubMed]

4. Zachariah, R.; Van Damme, W.; Arendt, V.; Schmit, J.C.; Harries, A.D. The HIV/AIDS epidemic in sub-Saharan Africa: Thinking ahead on programmatic tasks and related operational research. J. Int. AIDS Soc. 2011, 14, S7. [CrossRef] [PubMed]

5. Bain, L.E.; Nkoke, C.; Noubiap, J.J.N. UNAIDS 90-90-90 targets to end the AIDS epidemic by 2020 are not realistic: Comment on "Can the UNAIDS 90-90-90 target be achieved? A systematic analysis of national HIV treatment cascades". BMJ Glob. Health 2017, 2, e000227. [CrossRef] [PubMed]

6. Beccari, M.V.; Mogle, B.T.; Sidman, E.F.; Mastro, K.A.; Asiago-Reddy, E.; Kufel, W.D. Ibalizumab, a Novel Monoclonal Antibody for the Management of Multidrug-Resistant HIV-1 Infection. Antimicrob. Agents Chemother. 2019, 63. [CrossRef] [PubMed]

7. Spreen, W.R.; Margolis, D.A.; Pottage, J.C. Long-acting injectable antiretrovirals for HIV treatment and prevention. Curr. Opin. HIV AIDS 2013, 8, 565-571. [CrossRef] [PubMed] 
8. Riccardi, N.; Berruti, M.; Del Puente, F.; Taramasso, L.; Di Biagio, A. Ibalizumab and Fostemsavir in the Management of Heavily Pre-Treated HIV-infected Patients. Recent Patents Anti-Infect. Drug Discov. 2019, 13, 190-197. [CrossRef] [PubMed]

9. Nyaku, A.N.; Kelly, S.G.; Taiwo, B.O. Long-Acting Antiretrovirals: Where Are We Now? Curr. HIV/AIDS Rep. $2017,14,63-71$. [CrossRef] [PubMed]

10. Darbyshire, J.; Foulkes, M.; Peto, R.; Duncan, W.; Babiker, A.; Collins, R.; Hughes, M.; Peto, T.E.; Walker, S.A.; Walker, A. Zidovudine (AZT) versus AZT plus didanosine (ddI) versus AZT plus zalcitabine (ddC) in HIV infected adults. Cochrane Database Syst. Rev. 2000, CD002038. [CrossRef]

11. Bozzi, A.; D'Andrea, G.; Brisdelli, F. AZT: An old drug with new perspectives. Curr. Clin. Pharmacol. 2008, 3, 20-37. [CrossRef]

12. Durand-Gasselin, L.; Pruvost, A.; Dehée, A.; Vaudre, G.; Tabone, M.-D.; Grassi, J.; Leverger, G.; Garbarg-Chenon, A.; Bénech, H.; Dollfus, C. High Levels of Zidovudine (AZT) and Its Intracellular Phosphate Metabolites in AZT- and AZT-Lamivudine-Treated Newborns of Human Immunodeficiency Virus-Infected Mothers. Antimicrob. Agents Chemother. 2008, 52, 2555-2563. [CrossRef] [PubMed]

13. Guidelines for the Use of Antiretroviral Agents in Pediatric HIV Infection. Available online: https://aidsinfo.nih.gov/guidelines/ $\mathrm{html} / 2$ /pediatric-arv/510/antiretroviral-management-of-newborns-with-perinatal-hiv-exposure-or-perinatal-hiv (accessed on 30 June 2020).

14. Recommendations for the Use of Antiretroviral Drugs in Pregnant Women with HIV Infection and Interventions to Reduce Perinatal HIV Transmission in the United States. Available online: https://aidsinfo.nih.gov/guidelines/html/3/perinatal/180/ intrapartum-antiretroviral-therapy-prophylaxis (accessed on 30 June 2020).

15. DeLory, T.; Papot, E.; Rioux, C.; Charpentier, C.; Auge-Courtoi, C.; Michard, F.; Peytavin, G.; Descamps, D.; Matheron, S.; Yazdanpanah, Y. Foscarnet, zidovudine and dolutegravir combination efficacy and tolerability for late stage HIV salvage therapy: A case-series experience. J. Med. Virol. 2015, 88, 1204-1210. [CrossRef] [PubMed]

16. LaBonte, J.; Lebbos, J.; Kirkpatrick, P. Enfuvirtide. Nat. Rev. Drug Discov. 2003, 2, 345-346. [CrossRef] [PubMed]

17. Reynes, J.; Arastéh, K.; Clotet, B.; Cohen, C.; Cooper, D.A.; Delfraissy, J.F.; Eron, J.J.; Henry, K.; Katlama, C.; Kuritzkes, D.R.; et al. TORO: Ninety-six-week virologic and immunologic response and safety evaluation of enfuvirtide with an optimized background of antiretrovirals. AIDS Patient Care STDS 2007, 21, 533-543. [CrossRef] [PubMed]

18. Taramasso, L.; Dentone, C.; Alessandrini, A.; Bruzzone, B.; Icardi, G.; Garraffo, R.; De Macina, I.; Viscoli, C.; Di Biagio, A. HIV/HCV Collaborative Liguria Group Successful antiretroviral therapy by using unusual antiretroviral combinations in heavily pre-treated patients: Two case reports. Int. J. STD AIDS 2014, 26, 831-834. [CrossRef]

19. Viciana, P. Rilpivirine: The Key for Long-term Success. AIDS Rev. 2017, 19, 156-166. [CrossRef]

20. Pozniak, A.; Morales-Ramirez, J.; Katabira, E.; Steyn, D.; Lupo, S.H.; Santoscoy, M.; Grinsztejn, B.; Ruxrungtham, K.; Rimsky, L.T.; Vanveggel, S.; et al. Efficacy and safety of TMC278 in antiretroviral-naive HIV-1 patients: Week 96 results of a phase IIb randomized trial. AIDS 2010, 24, 55-65. [CrossRef]

21. Taramasso, L.; Di Biagio, A.; Riccardi, N.; Briano, F.; Di Filippo, E.; Comi, L.; Mora, S.; Giacomini, M.; Gori, A.; Maggiolo, F. Lipid profile changings after switching from rilpivirine/tenofovir disoproxil fumarate/emtricitabine to rilpivirine/tenofovir alafena-mide/emtricitabine: Different effects in patients with or without baseline hypercholesterolemia. PLoS ONE 2019, 11, $\mathrm{e} 0223181$.

22. van't Klooster, G.; Hoeben, E.; Borghys, H.; Looszova, A.; Bouche, M.P.; van Velsen, F.; Baert, L. Pharmacokinetics and disposition of rilpivirine (TMC278) nanosuspension as a long-acting injectable antiretroviral formulation. Antimicrob. Agents Chemother. 2010, 54, 2042-2050. [CrossRef]

23. Verloes, R.; Deleu, S.; Niemeijer, N.; Crauwels, H.; Meyvisch, P.; Williams, P. Safety, tolerability and pharmacokinetics of rilpivirine following administration of a long-acting formulation in healthy volunteers: RPV long-acting formulation: Safety and PK. HIV Med. 2015, 16, 477-484. [CrossRef]

24. Margolis, D.A.; Gonzalez-Garcia, J.; Stellbrink, H.J.; Eron, J.J.; Yazdanpanah, Y.; Podzamczer, D.; Lutz, T.; Angel, J.B.; Richmond, G.J.; Clotet, B.; et al. Safety, Efficacy and Durability of Long-acting Cabotegravir (CAB) and Rilpivirine (RPV) as Two-Drug IM Maintenance Therapy for HIV-1 Infection: LATTE-2 Week 160 Results Long-acting intramuscular cabotegravir and rilpivirine in adults with HIV-1 infection (LATTE-2): 96-week results of a randomised, open-label, phase 2b, non-inferiority trial. Lancet 2017, 390, 1499-1510. [PubMed]

25. Orkin, C.; Arasteh, K.; Hernández-Mora, M.G.; Pokrovsky, V.; Overton, E.T.; Girard, P.-M.; Oka, S.; Walmsley, S.; Bettacchi, C.; Brinson, C.; et al. Long-Acting Cabotegravir and Rilpivirine after Oral Induction for HIV-1 Infection. N. Engl. J. Med. 2020, 382, 1124-1135. [CrossRef]

26. Swindells, S.; Andrade-Villanueva, J.-F.; Richmond, G.J.; Rizzardini, G.; Baumgarten, A.; Masiá, M.; Latiff, G.; Pokrovsky, V.; Bredeek, F.; Smith, G.; et al. Long-Acting Cabotegravir and Rilpivirine for Maintenance of HIV-1 Suppression. N. Engl. J. Med. 2020, 382, 1112-1123. [CrossRef]

27. Bekker, L.-G.; Li, S.; Pathak, S.; Tolley, E.; Marzinke, M.; Justman, J.; Mgodi, N.; Chirenje, M.; Swaminathan, S.; Adeyeye, A.; et al. Safety and tolerability of injectable Rilpivirine LA in HPTN 076: A phase 2 HIV pre-exposure prophylaxis study in women. EClinicalMedicine 2020, 21, 100303. [CrossRef] [PubMed]

28. Whitfield, T.; Torkington, A.; Van Halsema, C. Profile of cabotegravir and its potential in the treatment and prevention of HIV-1 infection: Evidence to date. HIV/AIDS Res. Palliat. Care 2016, 8, 157-164. [CrossRef]

29. Kirby, T. Cabotegravir, a new option for PrEP. Lancet Infect. Dis. 2020, 20, 781. [CrossRef] 
30. Song, R.; Franco, D.; Kao, C.-Y.; Yu, F.; Huang, Y.; Ho, D.D. Epitope Mapping of Ibalizumab, a Humanized Anti-CD4 Monoclonal Antibody with Anti-HIV-1 Activity in Infected Patients. J. Virol. 2010, 84, 6935-6942. [CrossRef] [PubMed]

31. Emu, B.; Fessel, J.; Schrader, S.; Kumar, P.; Richmond, G.; Win, S.; Weinheimer, S.; Marsolais, C.; Lewis, S. Phase 3 Study of Ibalizumab for Multidrug-Resistant HIV-1. N. Engl. J. Med. 2018, 379, 645-654. [CrossRef]

32. Markham, A. Correction to: Ibalizumab: First Global Approval. Drugs 2018, 78, 859. [CrossRef]

33. Yant, S.R.; Mulato, A.; Stepan, G.; Villasenor, A.G.; Jin, D.; Margot, N.A.; Ahmadyar, S.; Ram, R.R.; Somoza, J.R.; Singer, E.; et al. GS-6207, a Potent and Selective First-in-Class Long-Acting HIV-1 Capsid Inhibitor. In Proceedings of the Conference on Retroviruses and Opportunistic Infections, Seattle, WA, USA, 4-7 March 2019. Abstract 480.

34. Daar, E.; McDonald, C.; Crofoot, G.; Ruane, P.; Sinclair, G.; De Jesus, E.; Berhe, M.; Ramgopal, M.; Patel, H.; Liu, Y.P.; et al. Dose-Response Relationship of Subcutaneous Long-Acting HIV Capsid Inhibitor GS-6207. In Proceedings of the Retro Conference on HIV and Opportunistic Infections, Boston, MA, USA, 8-11 March 2020. Poster 3691.

35. Chong, H.; Yao, X.; Zhang, C.; Cai, L.; Cui, S.; Wang, Y.; He, Y. Biophysical Property and Broad Anti-HIV Activity of Albuvirtide, a 3-Maleimimidopropionic Acid-Modified Peptide Fusion Inhibitor. PLoS ONE 2012, 7, e32599. [CrossRef]

36. Wu, H.; Yao, C.; Lu, R.J.; Zhang, T.; Wang, M.X.; Zhao, H.X.; Peng, H.Y.; Xie, Y.; Min, W.J.; Jiang, H.; et al. Albuvirtide, the first long-acting HIV-1 fusion inhibitor, suppressed viral replication in HIV-infected adults. In Proceedings of the Interscience Conference on Antimicrobial Agents and Chemotherapy (ICAAC), San Francisco, CA, USA, 9-12 September 2012. Paper H-554.

37. Zhang, H.-W.; Jin, R.; Yao, C.; Zhang, T.; Wang, M.-X.; Xia, W.; Peng, H.; Wang, X.; Lu, R.J.; Wang, C.; et al. Combination of long-acting HIV fusion inhibitor albuvirtide and LPV/r showed potent efficacy in HIV-1 patients. AIDS Res. Ther. 2016, 13, 8. [CrossRef] [PubMed]

38. Su, B.; Yao, C.; Zhao, Q.X.; Cai, W.P.; Wang, M.; Lu, H.Z.; Chen, Y.Y.; Liu, L.; Wang, H.; He, Y.; et al. Efficacy and safety of the long-acting fusion inhibitor al-buvirtide in antiretroviral-experienced adults with human immunodeficiency virus-1: Interim analysis of the randomized, controlled, phase 3, non-inferiority TALENT study. Chin. Med. J. (England) 2020, 133, $2919-2927$. [CrossRef] [PubMed]

39. Liu, Y.; Cao, W.; Sun, M.; Cao, W. Broadly neutralizing antibodies for HIV-1: Efficacies, challenges and opportunities. Emerg. Microbes Infect. 2020, 9, 194-206. [CrossRef]

40. Ghosn, J.; Pellegrin, I.; Goujard, C.; Deveau, C.; Viard, J.-P.; Galimand, J.; Harzic, M.; Tamalet, C.; Meyer, L.; Rouzioux, C.; et al. HIV-1 resistant strains acquired at the time of primary infection massively fuel the cellular reservoir and persist for lengthy periods of time. AIDS 2006, 20, 159-170. [CrossRef] [PubMed]

41. Chiodini, J. HIV drug interaction resources from the University of Liverpool. Travel Med. Infect. Dis. 2016, 14, 292-293. [CrossRef]

42. Prinapori, R.; Giannini, B.; Riccardi, N.; Bovis, F.; Giacomini, M.; Setti, M.; Viscoli, C.; Artioli, S.; Di Biagio, A. Predictors of retention in care in HIV-infected patients in a large hospital cohort in Italy. Epidemiol. Infect. 2018, 146, 606-611. [CrossRef] [PubMed]

43. Capetti, A.F.; De Socio, G.V.; Cossu, M.V.; Sterrantino, G.; Cenderello, G.; Cattelan, A.; Baldin, G.; Soria, A.; Riccardi, N.; Niero, F.P.; et al. Durability of dolutegravir plus boosted darunavir as salvage or simplification of salvage regimens in HIV-1 infected, highly treatment-experienced subjects. HIV Clin. Trials 2018, 19, 242-248. [CrossRef] 